\title{
Thin-film and single-crystal transistors based on a trifluoromethyl-substituted alternating thiophene/phenylene co-oligomer
}

Musubu Ichikawa ${ }^{1,2}$, Tatsuya Kato ${ }^{1}$, Tetsuro Uchino ${ }^{1}$, Takeo Tsuzuki ${ }^{1}$, Masamitsu Inoue ${ }^{1}$, Hyeon-Gu Jeon $^{1}$, Toshiki Koyama ${ }^{1}$, Yoshio Taniguchi ${ }^{1}$

${ }^{1}$ Functional Polymer Science Course, Division of Chemistry and Materials, Faculty of Textile Science and Technology, Shinshu University, 3-15-1 Tokida, Ueda 386-8567, Japan

${ }^{2}$ Presto, Japan Science and Technology Agency (JST), 4-8-1 Honcho, Kawaguchi, Saitama 332-0012, Japan

\begin{abstract}
We demonstrated the performance of thin-film transistors (TFTs) and single-crystal field-effect transistors (FETs) based on a trifluoromethyl-substituted alternating (thiophene/phenylene)-co-oligomer (AC5-CF3), 1,4-bis(5'-(4"'-trifluoromethylphenyl)thiophene-2'-yl)benzene. An FET with a fine AC5-CF3 single crystal demonstrated field-effect mobility as high as $3.1 \mathrm{~cm}^{2} \mathrm{~V}^{-1} \mathrm{~s}^{-1}$. This value implies that AC5-CF3 must be a useful n-type organic semiconducting material. The performance of AC5-CF3 TFTs depended on the substrate temperatures at which AC5-CF3 thin films were
\end{abstract}


deposited. From the viewpoint of mobility, threshold voltage and sub-threshold slope, we obtained the highest performance at the substrate temperature of $100^{\circ} \mathrm{C}$. This was because a higher substrate temperature for deposition enlarged the size of grains in $\mathrm{AC}-\mathrm{CF} 3$ thin films and improved the characteristics of grain boundaries. However, $120^{\circ} \mathrm{C}$ depositions of $\mathrm{AC} 5-\mathrm{CF} 3$ induced deep valley-like cracks in the thin films, probably because of the difference between the coefficients of thermal expansion for AC5-CF3 thin films and silicon wafer substrates, resulting in effects such as worsening mobility. AC5-CF3 TFTs prepared at $100^{\circ} \mathrm{C}$ deposition showed no channel length dependence of the field-effect mobility, and their average field-effect mobility was $0.55 \pm 0.05 \mathrm{~cm}^{2} \mathrm{~V}^{-1} \mathrm{~s}^{-1}$

Keywords: organic thin-film transistors, organic field-effect transistors, n-type semiconductors, alternating co-oligomer, (thiophene/phenylene)-co-oligomer, electron mobility

*Corresponding author at: Functional Polymer Science Course, Division of Chemistry and Materials, Faculty of Textile Science and Technology, Shinshu University, 3-15-1 Tokida, Ueda City, Nagano 386-8567, Japan

Tel: +81-268-21-5498; Fax: +81-268-21-5417

Email address: musubu@shinshu-u.ac.jp 


\section{Introduction}

Organic thin-film transistors (TFTs) have attracted considerable interest in recent years because of their numerous potential applications as flexible, large-area and low-cost electronic devices $[1,2]$. Among the many organic semiconductors investigated, pentacene has shown the highest mobility in a thin-film state, and this mobility is much higher than that of the hydrogenated amorphous silicon [3-5]. Most organic materials with high mobility in the thin-film state, such as pentacene and thiophene derivatives, are p-type semiconductors whose main carrier is a hole [6-9]. On the other hand, there have been few reports on high performance n-type organic TFTs [10-19]. To move ahead with organic electronics, it is required that complementary metal-oxide-semiconductor (CMOS)-like logic circuits that call for both $\mathrm{p}$ - and n-type TFTs be used as they consume less power and have simple circuit designs. Thus, performance improvement of n-type organic TFT is a priority to this end.

There have been some reports about good n-type organic transistor materials that are based on trifluoromethyl substitution $[15,20,21]$. We also thought that $\mathrm{CF}_{3}$-substituted materials become good n-type organic FET materials [22], and synthesized AC5-CF3 as shown in the Fig 1(a) based on a report by Katz et al. [23]. In that article, the authors reported that a material similar to $\mathrm{AC} 5-\mathrm{CF} 3$ but without a $\mathrm{CF}_{3}$ group worked as a p-type FET material with a field-effect hole mobility of $0.04 \mathrm{~cm}^{2} / \mathrm{Vs}$ at best. Here, we demonstrate that AC5-CF3 could be a potential candidate for organic n-type semiconductors that have high electron mobility. Note that Hotta et 
al. recently reported the synthesis of the same material [24], formation of its crystal [25], the crystal structure and its field-effect transistors (FETs) [26]. However, Hotta et al. reported that AC-CF3 single-crystal FETs showed n-type behavior and that the electron mobility of the FETs ranged from 1.6 to $3.9 \times 10^{-2} \mathrm{~cm}^{2} \mathrm{~V}^{-1} \mathrm{~s}^{-1}$. Here, we report the performance of optimally fabricated AC5-CF3 TFTs and single-crystal FETs, and demonstrate that AC-CF3 will be able to be utilised as an n-type organic transistor material with high mobility.

\section{Material and Methods}

\subsection{Material synthesis and characterisation}

AC5-CF3 was synthesized by the Stille coupling reaction of 4-trifluoromethyl benzene with the corresponding stannyl reagent in the presence of $\mathrm{Pd}_{2}$ (trans, trans-dibenzylidene acetone $)_{3}$ and (tert-butyl) ${ }_{3} \mathrm{P}$ in dimethylformamide with microwave heating. The synthetic root was slightly different as per a report by Katagiri et al. [24]. We confirmed its structure by matrix-assisted laser desorption/ionization (MALDI) time-of-flight (TOF) mass spectroscopy (Perspective Biosystems, Voyager-DE PRO) and ${ }^{1} \mathrm{H}$ NMR (Bruker AVANCE 400)). We used the material after sublimating it in a pure argon gas flow, and prepared AC5-CF3 single crystals from its vapour in a chamber filled with nitrogen adding 10 vol\% hydrogen. Details of this method have been described elsewhere [27].

The ionization potential (Ip) of AC5-CF3 was determined by photoelectron emission 
yield spectroscopy with Riken-Keiki AC-2 at an irradiation light power of $10 \mathrm{nW} \mathrm{cm}^{-2}$. The sample films of the organic materials were prepared by vacuum evaporation in the same conditions as can be used to fabricate TFTs on glass substrates whose surface was covered with indium thin oxide to prevent charging. We determined the lowest unoccupied molecular orbital (LUMO) level of AC5-CF3 from the difference between Ip and the band gap that was estimated from the absorption edge of 100-nm-thick AC5-CF3 thin film deposited on a quartz substrate.

X-ray diffraction (XRD) patterns were measured with a Rigaku Rotaflex using $\mathrm{Cu} \mathrm{K \alpha}$ radiation at $\theta / 2 \theta$ geometry. The sample films, all with thicknesses $100 \mathrm{~nm}$, were evaporated onto silicon wafers with $\mathrm{SiO}_{2}$ in the same conditions as can be used for TFT fabrication. The X-ray was generated from a $150-\mathrm{mA}$ electron beam accelerated at $40 \mathrm{kV}$. The crystal structure of AC5-CF3 was analyzed with a Bruker-AXS APEX II. We prepared the crystal for analysis from the vapour phase.

\subsection{Device fabrications and measurements}

AC5-CF3 FETs with a single crystal of AC5-CF3 were fabricated as follows. A crystal was placed on a silicon wafer that had a 400-nm-thick thermally grown silicon dioxide layer as a gate insulator. Without treating the surface of the gate insulator, we simply washed the substrates with 2-propanol. After placing the crystal, we deposited gold electrodes on the source and drain electrodes on the crystal through a shadow mask. Channel length and width of the transistors 
were 25 and $200 \mu \mathrm{m}$, respectively.

We also prepared thin-film transistors using AC5-CF3 by depositing AC5-CF3 on a silicon wafer with a 200-nm-thick $\mathrm{SiO}_{2}$ layer through thermal evaporation. We kept temperature of the substrates at a temperature in a range from room temperature to $120^{\circ} \mathrm{C}$ during the deposition. The thickness of AC5-CF3 thin film was $60 \mathrm{~nm}$. Finally, we prepared source and drain electrodes on the thin film by thermal evaporation of gold. Channels of the thin-film transistors were made with another shadow mask. We varied the channel length of the thin-film transistors from 30 to $100 \mu \mathrm{m}$, and kept the width of all the TFTs constant at $2000 \mu \mathrm{m}$. An Agilent semiconductor parameter analyzer (B1500A) or a pair of two Keithley source meters (2410) recorded characteristics of the transistors in a probe station (Measure Jig, MJ-10) under vacuum at room temperature.

\section{Results and Discussion}

Figure 1(c) shows a photograph of AC5-CF3 single-crystal FET prepared by fixing a single crystal of AC5-CF3 on silicon wafer, which has a thermally grown 400-nm-thick silicon dioxide layer, as a gate insulator. Figure 1(b) shows the schematic cross-sectional structure of the FET; the configuration of the electrodes is the so-called top contact and bottom gate configuration. The crystal seems to uniformly contact the insulator as there were no obvious voids or defects in the area of the crystal seen in the photograph, and hence, the FET device should work well. 
Figure 2(a) shows drain current versus drain voltage $\left(I_{D^{-}} V_{D}\right)$ characteristics of AC5-CF3 single-crystal FET at several different gate voltages $\left(V_{G}\right)$. As clearly shown in the figure, the device works as an n-type FET, and this means that AC5-CF3 single crystal is an n-type semiconducting material in which electrons behave as charge carriers. The drain current at a low $V_{D}$ region (below $20 \mathrm{~V}$ ) was limited, and thus we could not observe the linear behavior. The current limitation at the low $V_{D}$ region probably resulted from the configuration of the source and drain electrodes for the device. In short, similar current limitations are frequently observed in the case of the so-called top contact FETs in general, and/or the LUMO level of AC5-CF3 at $3.1 \mathrm{eV}$ [28] results in a slightly large electron injection barrier from the gold source electrode to the semiconductor. However, the $I_{D^{-}} V_{D}$ characteristics exhibited well-defined saturation behavior. Figure 2(b) shows $I_{D^{-}} V_{G}$ and the square root of $I_{D^{-}} V_{G}$ characteristic of the FET at a drain voltage of $200 \mathrm{~V}$. We obtained the field-effect mobility $\left(\mu_{F E}\right)$ of an electron in AC5-CF3 single-crystal FET from saturated drain currents $\left(I_{D, \text { sat }}\right)$ based on Fig. 2(b) using the following equation: [29]

$$
I_{D, s a t}=W / 2 L C_{i} \mu_{F E}\left(V_{G}-V_{T}\right)^{2}
$$

where $W$ and $L$ are width and length of the channel, respectively, and $C_{i}$ and $V_{T}$ are specific capacitance of the gate dielectric and the threshold voltage, respectively. The obtained mobility was as high as $3.1 \mathrm{~cm}^{2} \mathrm{~V}^{-1} \mathrm{~s}^{-1}$. The field-effect mobility of electrons of $3.1 \mathrm{~cm}^{2} \mathrm{~V}^{-1} \mathrm{~s}^{-1}$ is higher compared to other highly mobile electrons in organic semiconductors that have been already reported [17, 18, 30]. This means that AC5-CF3 should be a potential candidate for n-type 
organic transistors. Note that the high operation voltage of $200 \mathrm{~V}$ probably is a result of the rather thick $(400 \mathrm{~nm}) \mathrm{SiO}_{2}$ layer as gate insulator.

Figure 3(a) shows XRD pattern of the FET with mobility of $3.1 \mathrm{~cm}^{2} \mathrm{~V}^{-1} \mathrm{~s}^{-1}$. We can observe several peaks whose width is very narrow; the full width at half maximum (FWHM) of a peak at $2 \theta$ of 10.76 degree is only 0.09 degree. All the peaks result from a reflection by the $c$-axis; they are first-order and other higher-order diffractions as assigned in the figure. This indicates that the crystal is of high quality and on the substrate in which the normal direction corresponds with the direction of the $c$-axis. As shown in Fig. 4, the molecular interactions due to $\pi$-electrons appear to be stronger in the $a b$ plane than in other planes, and consequently, charge carriers move smoothly in the $a b$ plane. The high quality of the crystal also enhances the carrier transport in the crystal. As a result, the FET device showed a high electron mobility, which was larger than twice of a mobility in a very recent report on AC5-CF3 single-crystal FETs.[31]

We demonstrated above that AC5-CF3 is a potential candidate for high mobility n-type organic semiconductors for transistors from the results of the AC5-CF3 single-crystal FET. However, mobility in thin-film states not a single-crystalline state should be more important from the viewpoint of practical use because organic transistors have received a lot of interests in the field of large area and/or flexible electronic devices. Consequently, we describe the characteristics of thin-film transistors based on AC5-CF3 in the following paragraphs. Figure 5(a) shows an example curve set of the output characteristic of AC5-CF3 thin-film transistor, where 
we deposited AC5-CF3 on the substrate at a temperature of $100^{\circ} \mathrm{C}$. Here, we employed Si wafers with a thin $(200 \mathrm{~nm}) \mathrm{SiO}_{2}$ layer to reduce the operation voltage. The figure clearly shows that the device worked as an n-type TFT. Figure 5(b) shows the transfer curve of TFT and also shows the square root of $I_{D}$ versus $V_{G}$ plot at a drain voltage of $100 \mathrm{~V}$. We determined the field-effect mobility of the device from the slope of the root $I_{D}$ versus $V_{G}$ curve using Eq. (1); the mobility was $0.60 \mathrm{~cm}^{2} \mathrm{~V}^{-1} \mathrm{~s}^{-1}$. We fabricated six TFTs under the same condition, and the average mobility was $0.55 \pm 0.05 \mathrm{~cm}^{2} \mathrm{~V}^{-1} \mathrm{~s}^{-1}$. While this mobility is obviously lower than that of the FET based on a single crystal, the mobility is sufficiently large, as large as n-type organic TFT materials [15, 17].

Figure 6 shows field-effect electron mobility, threshold voltages and sub-threshold slopes (SS) of TFTs with AC5-CF3 thin films deposited at different substrate temperatures. The figure clearly indicates certain tendencies: mobility increased and threshold voltage and SS decreased with increasing substrate temperature, while all the parameters became worse at a temperature of $120^{\circ} \mathrm{C}$. Figure 7 shows the set of surface morphologies of $\mathrm{AC}-\mathrm{CF} 3$ thin films prepared at different substrate temperatures. The morphological change shown in the figure is sufficiently consistent with the temperature dependences of mobility and threshold voltage. In short, the grain sizes of AC5-CF3 thin films became large with increasing substrate temperature. We believe that increasing the grain sizes directly and beneficially influences mobility. As shown in Fig. 7(c), however, some deep valleys that seemed to segment adjacent grains were observed. 
Although we are unsure about the appearance of the valleys on the film, we speculate that the valleys appear because of a difference in the coefficients of thermal expansion (CTE) of AC5-CF3 thin films and silicon wafers because CTEs of organic materials are generally much larger than $\mathrm{Si}$ whose linear CTE is only $4 \times 10^{-6} \mathrm{~K}^{-1}$. The valleys probably interfered with electron transport of inter-grains, resulting in reduced mobility at $120^{\circ} \mathrm{C}$. Note that clear step-terrace structures appeared on all the images and the height of the steps corresponded to the length of the $a$-axis of the unit cell of AC5-CF3.

Furthermore, the threshold voltage decreased from the improved quality of AC5-CF3 thin film induced by temperature-controlled deposition. Enlarging grains at higher temperature depositions may be accompanied by qualitative improvement of thin films such as fewer defects that work as charge carrier traps. XRD patterns from AC5-CF3 thin films prepared at different temperatures shown in Fig. 3 support the qualitative improvement of the thin films. In short, the change of threshold voltage shown in Fig. 6 can be consistent with this improvement. Figure 8 shows the channel length dependence of the field-effect mobility of AC5-CF3 TFTs prepared at different deposition temperatures. In case of the room temperature deposition of AC5-CF3, the field-effect mobility of TFTs slightly decreased with increasing channel lengths. In contrast, the mobility showed no dependence on the channel length in the case of $100^{\circ} \mathrm{C}$ deposition of AC5-CF3. According to the atomic force microscope (AFM) observation shown in Fig. 7, the size of the grains is much smaller than the channel length of $30 \mu \mathrm{m}$ even in the case of $100^{\circ} \mathrm{C}$ 
deposition of AC5-CF3. Consequently, the independence of the mobility from the channel length observed in the case of the $100^{\circ} \mathrm{C}$ deposition indicates that the deposition at higher temperature probably improves the quality of not only grains but also boundaries of grains. We can see from the AFM image shown in Fig. 7(b) that the grains with the size of about $1-3 \mu \mathrm{m}$ tightly pack. However, the mobility of the TFTs with $120^{\circ} \mathrm{C}$ depositions also decreased with increasing channel length. This phenomenon occurs because of the presence of the valleys observed in the film (see Fig. 7(a)).

\section{Summary}

We demonstrated the performance of optimally fabricated AC5-CF3 TFTs and its single-crystal FET. AC5-CF3 single-crystal FET showed field-effect mobility as high as $3.1 \mathrm{~cm}^{2}$ $\mathrm{V}^{-1} \mathrm{~s}^{-1}$. This value implies that AC5-CF3 must be a useful n-type organic semiconducting material. We also prepared AC5-CF3 TFTs, and the performance of the TFTs depended on the substrate temperatures during AC5-CF3 deposition. We obtained the highest performance from the viewpoint of mobility, threshold voltage and sub-threshold slope when AC5-CF3 thin films were deposited at the substrate temperature of $100^{\circ} \mathrm{C}$. This was because a higher substrate temperature for the deposition enlarged the size of grains of AC-CF3 and improved the characteristics of grain boundaries. However, the $120^{\circ} \mathrm{C}$ deposition induced deep valleys such as cracks and worsening mobility. AC5-CF3 TFTs prepared at $100^{\circ} \mathrm{C}$ depositions showed no channel 
length dependence of the field-effect mobility. This is a very useful property for practical applications.

Acknowledgements: This work was supported by Regional Innovation Cluster Program of Nagano, granted by MEXT, Japan. This article is dedicated to the first principal of the Faculty of Textile Science and Technology, Shinshu University, Chotaro Harizuka, on the occasion of the 100th anniversary of the faculty. 


\section{References and Notes}

[1] C. D. Dimitrakopoulos, P. R. L. Malenfant, Adv. Mater. 14 (2002) 99.

[2] S. R. Forrest, Nature 428 (2004) 911.

[3] S. F. Nelson, Y. Y. Lin, D. J. Gundlach, T. N. Jackson, Appl. Phys. Lett. 72 (1998) 1854.

[4] H. Klauk, M. Halik, U. Zschieschang, G. Schmid, W. Radlik, W. Weber, J. Appl. Phys. $92(2002) 5259$.

[5] T. W. Kelley, L. D. Boardman, T. D. Dunbar, D. V. Muyres, M. J. Pellerite, T. P. Smith, J. Phys. Chem. B 107 (2003) 5877.

[6] M. Ichikawa, H. Yanagi, Y. Shimizu, S. Hotta, N. Suganuma, T. Koyama, Y. Taniguchi, Adv. Mater. 14 (2002) 1272.

[7] H. Meng, J. Zheng, A. J. Lovinger, B.-C. Wang, P. G. V. Patten, Z. Bao, Chem. Mater. 15 (2003) 1778.

[8] M. Halik, H. Klauk, U. Zschieschang, G. Schmid, W. Radlik, S. Ponomarenko, S. Kirchmeyer, W. Weber, J. Appl. Phys. 93 (2003) 2977.

[9] T. Yamamoto, K. Takimiya, J. Am. Chem. Soc. 129 (2007) 2224.

[10] C. R. Newman, C. D. Frisbie, D. A. d. S. Filho, J.-L. Bredas, P. C. Ewbank, K. R. Mann, Chem. Mater. 16 (2004) 4436.

[11] Z. Bao, A. J. Lovinger, J. Brown, J. Am. Chem. Soc. 120 (1998) 207.

[12] H. E. Katz, A. J. Lovinger, J. Johnson, C. Kloc, T. Siegrist, W. Li, Y.-Y. Lin, A. 
Dodabalapur, Nature 404 (2000) 478.

[13] P. R. L. Malenfant, C. D. Dimitrakopoulos, J. D. Gelorme, L. L. Kosbar, T. O. Graham, Appl. Phys. Lett. 80 (2002) 2517.

[14] A. Facchetti, M.-H. Yoon, C. L. Stern, H. E. Katz, T. J. Marks, Angew. Chem. Int. Ed. 42 (2003) 3900.

[15] S. Ando, R. Murakami, J.-i. Nishida, H. Tada, Y. Inoue, S. Tokito, Y. Yamashita, J. Am. Chem. Soc. 127 (2005) 14996.

[16] N. J. Haddock, B. Domercq, B. Kippelen, Electron Lett 41 (2005) 444.

[17] S. Tatemichi, M. Ichikawa, T. Koyama, Y. Taniguchi, Appl. Phys. Lett. 89 (2006) 112108.

[18] D. Shukla, S. F. Nelson, D. C. Freeman, M. Rajeswaran, W. G. Ahearn, D. M. Meyer, J. T. Carey, Chem. Mater. 20 (2008) 7486.

[19] M. Chikamatsu, A. Itakura, Y. Yoshida, R. Azumi, K. Yase, Chem. Mater. 20 (2008) 7365.

[20] M. Mamada, J.-i. Nishida, D. Kumaki, S. Tokito, Y. Yamashita, Chem. Mater. 19 (2007) 5404.

[21] C.-a. Di, J. Li, G. Yu, Y. Xiao, Y. Guo, Y. Liu, X. Qian, D. Zhu, Organic Letters 10 (2008) 3025 .

[22] S. Ando, J.-i. Nishida, H. Tada, Y. Inoue, S. Tokito, Y. Yamashita, J. Am. Chem. Soc. 127 
(2005) 5336.

[23] X. M. Hong, H. E. Katz, A. J. Lovinger, B.-C. Wang, K. Raghavachari, Chem. Mater. 13 (2001) 4686.

[24] T. Katagiri, S. Ota, T. Ohira, T. Yamao, S. Hotta, J Heterocyclic Chem. 44 (2007) 853.

[25] T. Yamao, T. Miki, H. Akagami, Y. Nishimoto, S. Ota, S. Hotta, Chem. Mater. 19 (2007) 3748.

[26] S. Hotta, Y. Shimizu, T. Yamao, M. Goto, R. Azumi, Chem. Lett. 38 (2009) 294.

[27] M. Ichikawa, R. Hibino, M. Inoue, T. Haritani, S. Hotta, T. Koyama, Y. Taniguchi, Adv. Mater. 15 (2003) 213.

[28] Ip and bandgap of AC5-CF3 were 5.8 and $2.7 \mathrm{eV}$, respectively.

[29] S. M. Sze, Physics of semiconductor devices. 2nd ed.; John Wiley \& Sons: New York, 1981.

[30] X. H. Zhang, B. Kippelen, Appl. Phys. Lett. 93 (2008) 133305.

[31] T. Yamao, Y. Shimizu, H. Kuriki, T. Katagiri, S. Hotta, Jpn J. Appl. Phys. 49 (2010) $01 \mathrm{AB} 01$. 


\section{Figure Captions}

Figure 1. (a) Chemical structure of AC5-CF3, (b) Schematic cross-sectional structure and (c) example top-view photograph of FET with an AC5-CF3 single crystal.

Figure 2. (a) $I_{D^{-}} V_{D}$ and (b) $I_{D^{-}} V_{G}$ characteristics of AC5-CF3 single-crystal FET. We measured the $I_{D^{-}} V_{G}$ curves at $V_{D}$ of $200 \mathrm{~V}$.

Figure 3. (a) X-ray diffraction pattern of AC5-CF3 single crystal that was used for the FET with electron mobility of $3.1 \mathrm{~cm}^{2} \mathrm{~V}^{-1} \mathrm{~s}^{-1}$. X-ray diffraction patterns of AC5-CF3 thin films deposited at different substrate temperatures.

Figure 4. Crystal structure of AC5-CF3. Images on the left and right are side and top views, respectively. Crystal structure is as follows: Crystal system: monoclinic; space group: P2 ${ }_{1} / \mathrm{c}$; number of molecules in cell $=2$; cell parameters: $a=25.070(9) \AA, b=7.354(3) \AA, c=5.742(2) \AA$, $\alpha=\gamma=90^{\circ}$ and $\beta=94.327(5)^{\circ}$. CCDC 780290 contains the supplementary crystallographic data for this paper. These data can be obtained free of charge from The Cambridge Crystallographic Data Centre via www.ccdc.cam.ac.uk/data_request/cif.

Figure 5. (a) $I_{D^{-}} V_{D}$ and (b) $I_{D^{-}} V_{G}$ characteristics of AC5-CF3 thin-film transistor of channel 
length $50 \mu \mathrm{m}$, where AC5-CF3 thin film was deposited at a substrate temperature of $100^{\circ} \mathrm{C}$.

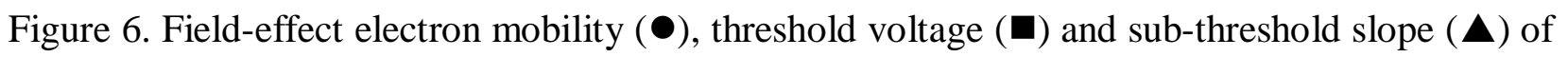
AC5-CF3 thin-film transistors (channel length of $50 \mu \mathrm{m}$ ) as a function of substrate temperature during the deposition of AC5-CF3.

Figure 7. Surface morphologies of AC5-CF3 thin films deposited at different substrate temperatures: (a) room temperature, (b) $100^{\circ} \mathrm{C}$ and (c) $120^{\circ} \mathrm{C}$. Vertically long color bars on the right of each image indicate height scale in nanometres.

Figure 8. Channel length dependence of field-effect mobility of AC5-CF3 thin-film transistors prepared at different substrate temperatures for AC5-CF3 depositions. 
(a)

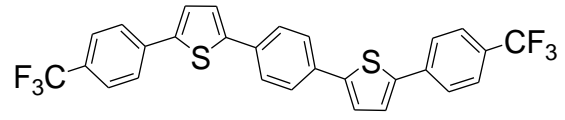

(b)

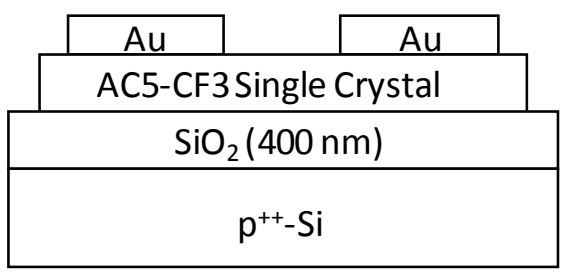

(c)

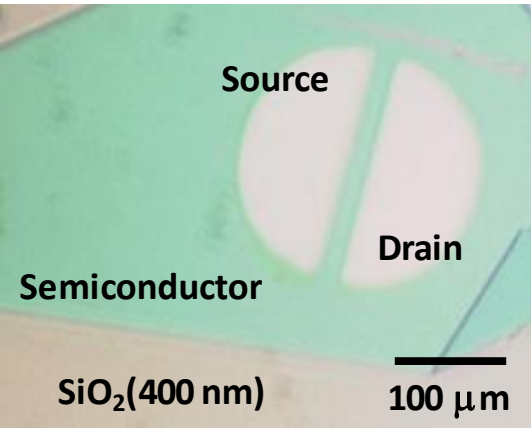

Figure 1. (a) Chemical structure of AC5-CF3, (b) Schematic cross-sectional structure and (c) example top-view photograph of FET with an AC5-CF3 single crystal. 

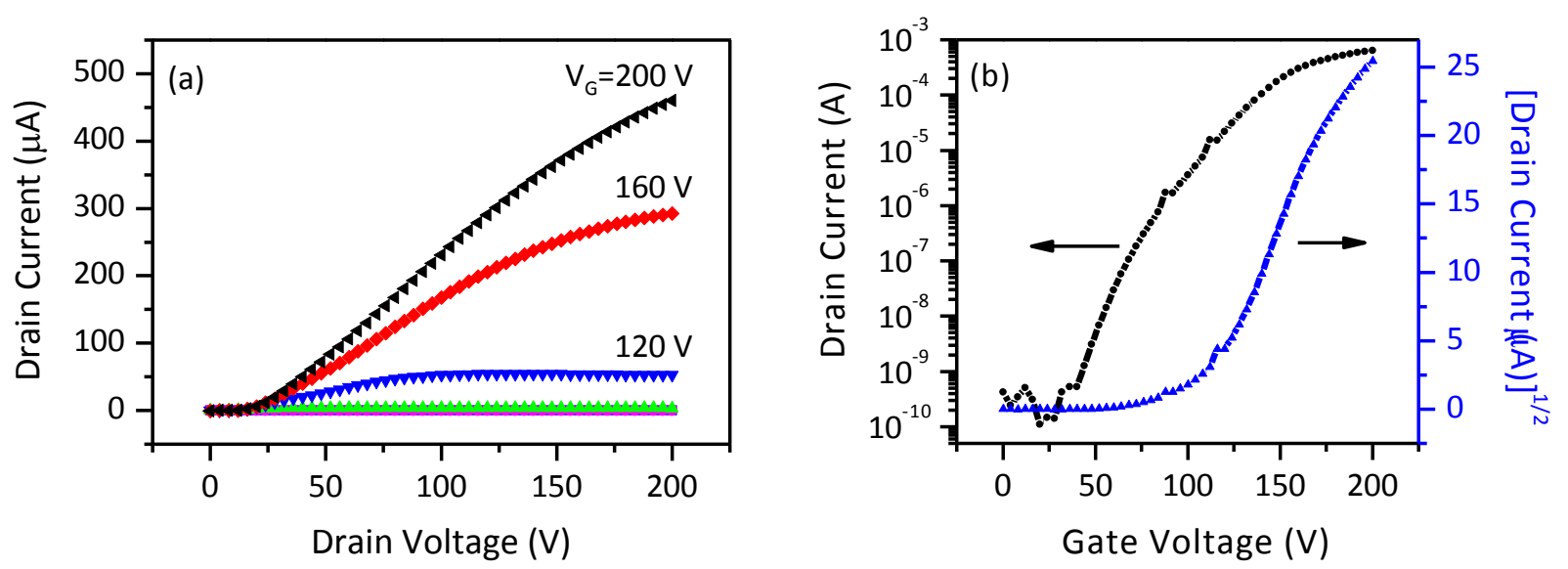

Figure 2. (a) $I_{D^{-}} V_{D}$ (a) and (b) $I_{D^{-}} V_{G}$ characteristics of AC5-CF3 single-crystal FET. We measured the $I_{D^{-}} V_{G}$ curves at $V_{D}$ of $200 \mathrm{~V}$. 


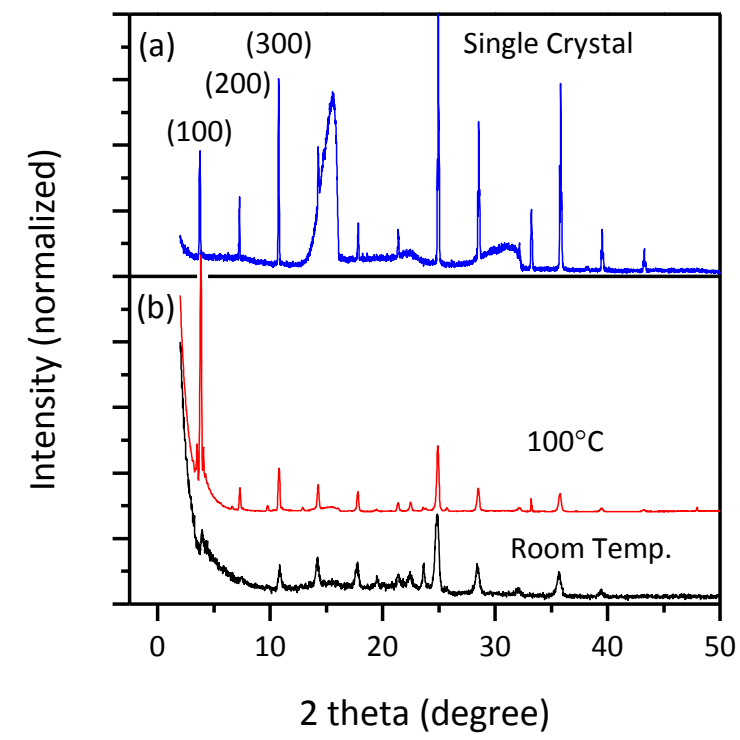

Figure 3. (a) X-ray diffraction pattern of AC5-CF3 single crystal that was used for the FET with electron mobility of $3.1 \mathrm{~cm}^{2} \mathrm{~V}^{-1} \mathrm{~s}^{-1}$. (b) X-ray diffraction patterns of AC5-CF3 thin films deposited at different substrate temperatures. 


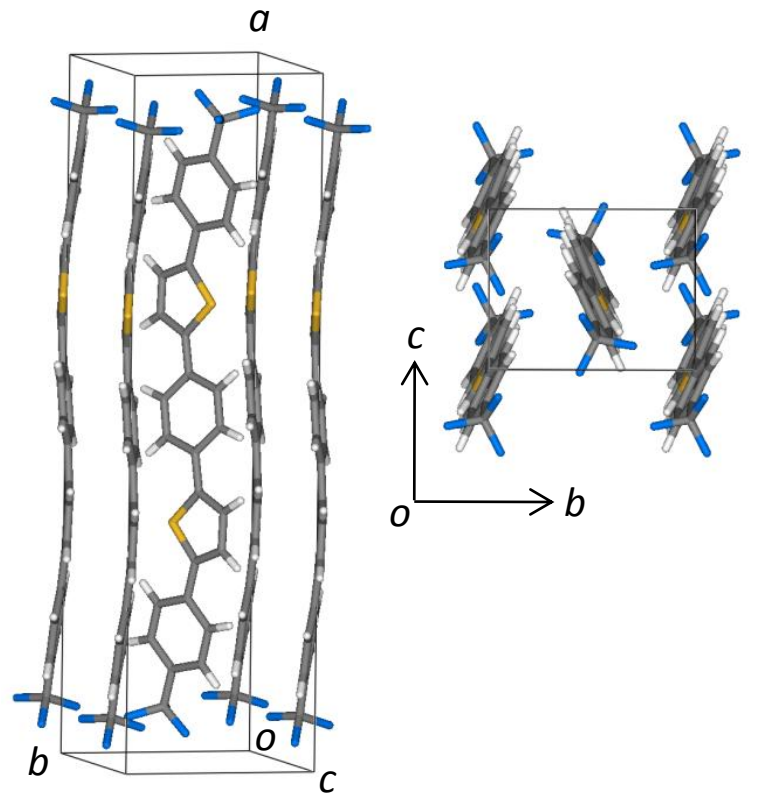

Figure 4. Crystal structure of AC5-CF3. Images on the left and right are side and top views, respectively. Crystal structure is as follows. Crystal system: monoclinic; space group: $\mathrm{P} 2{ }_{1} / \mathrm{c}$; number of molecules in cell: 2 ; cell parameters: $a=25.070(9) \AA, b=7.354(3) \AA, c=5.742(2) \AA$, $\alpha=\gamma=90^{\circ}$ and $\beta=94.327(5)^{\circ} . \mathrm{CCDC} 780290$ contains the supplementary crystallographic data for this paper. These data can be obtained free of charge from The Cambridge Crystallographic Data Centre via www.ccdc.cam.ac.uk/data_request/cif. 

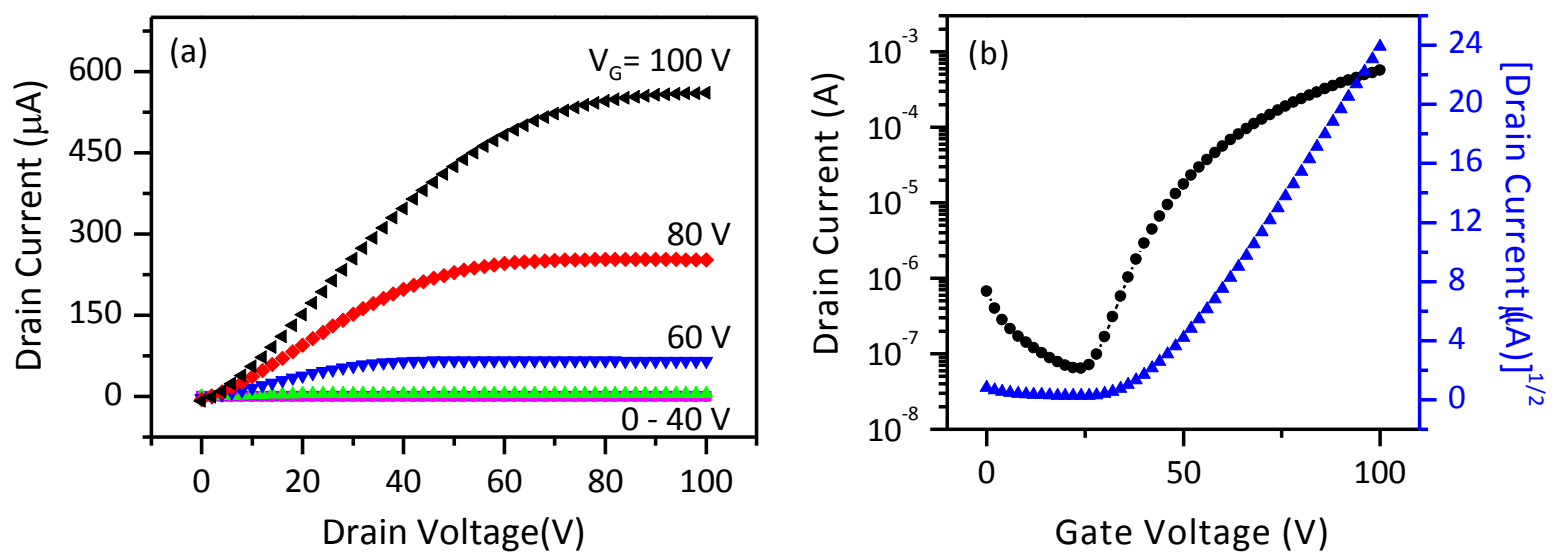

Figure 5. (a) $I_{D^{-}} V_{D}$ and (b) $I_{D^{-}} V_{G}$ characteristics of AC5-CF3 thin-film transistor whose channel length is $50 \mu \mathrm{m}$, where AC5-CF3 thin film was deposited at a substrate temperature of $100^{\circ} \mathrm{C}$. 


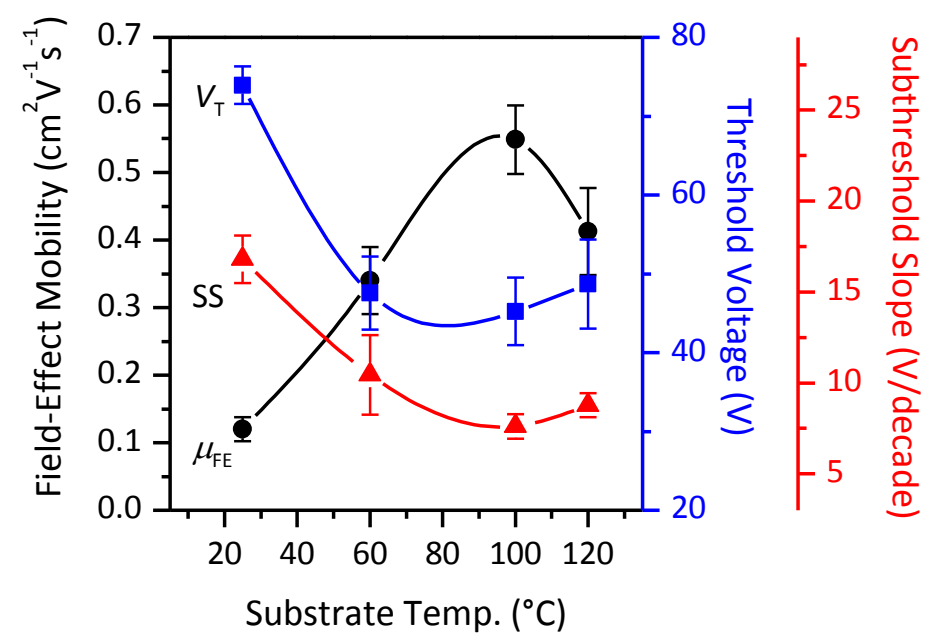

Figure 6. Field-effect electron mobility $(\bullet)$, threshold voltage $(\boldsymbol{\square})$ and sub-threshold slope $(\boldsymbol{\Delta})$ of AC5-CF3 thin-film transistors (channel length of $50 \mu \mathrm{m}$ ) as a function of substrate temperature during the deposition of AC5-CF3. 

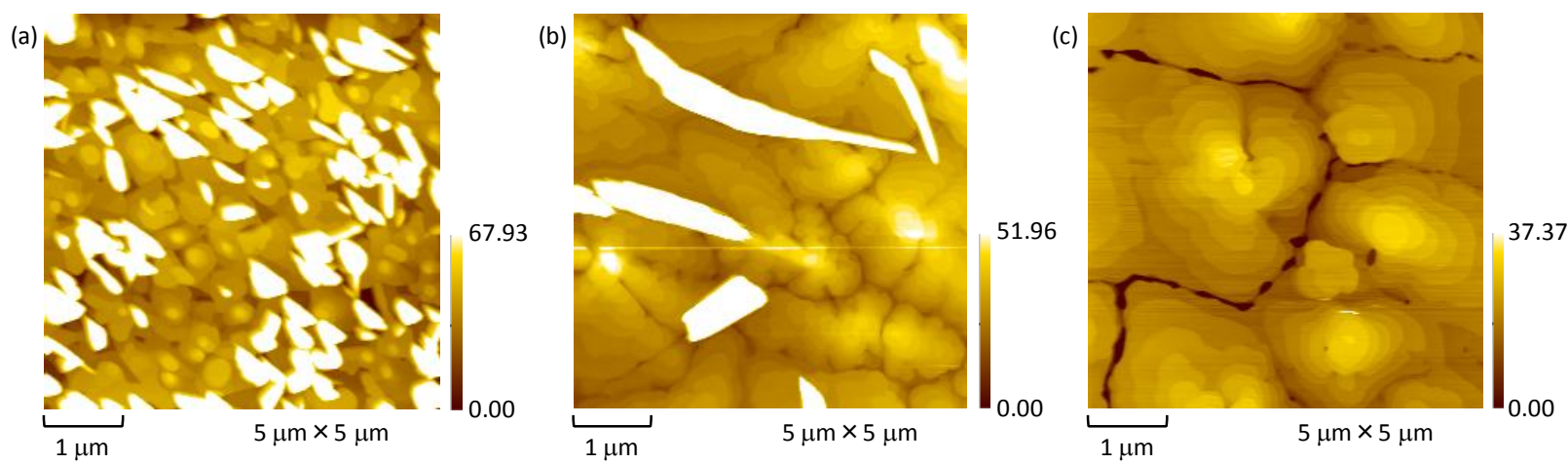

Figure 7. Surface morphologies of AC5-CF3 thin films deposited at different substrate temperatures: (a) room temperature, (b) $100^{\circ} \mathrm{C}$ and (c) $120^{\circ} \mathrm{C}$. The vertically long, color bars on the right of each image mean height scale in nanometres. 


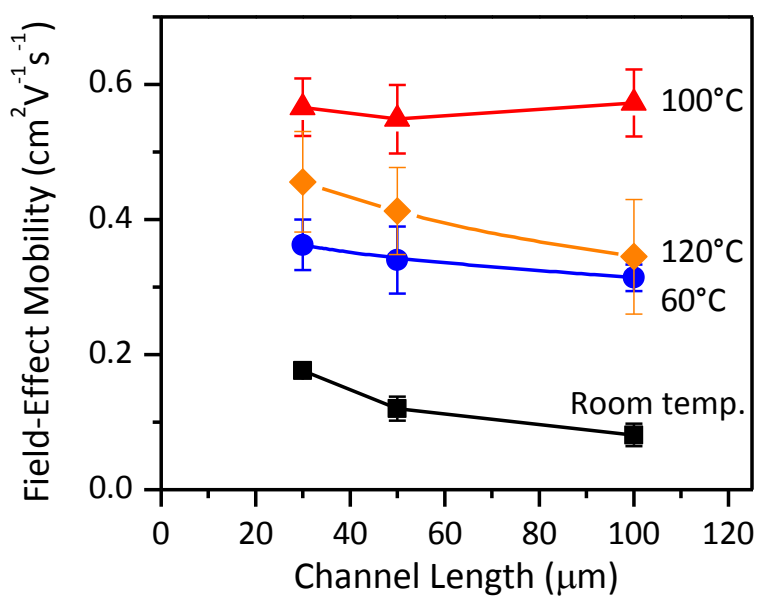

Figure 8. Channel length dependence of the field-effect mobility of AC5-CF3 thin-film transistors prepared at different substrate temperatures for AC5-CF3 depositions. 